\title{
Edoxaban zur Schlaganfallprophylaxe bei Patienten mit nicht-valvulärem Vorhofflimmern und zur Behandlung venöser Thromboembolien: ein interdisziplinäres Positionspapier
}

\author{
Thomas W. Weiss · Miklos Rohla (D) - Benjamin Dieplinger · Hans Domanovits · Dietmar Fries • Milan R. Vosko • \\ Thomas Gary · Cihan Ay
}

Eingegangen: 10. August 2016 / Angenommen: 2. Februar 2017 / Online publiziert: 24. Februar 2017

(c) Der/die Autor(en) 2017. Dieser Artikel ist eine Open-Access-Publikation.

\begin{abstract}
Zusammenfassung Mit Edoxaban ist der nun neueste Vertreter der Nicht-Vitamin K Antagonisten Oralen Antikoagulanzien (NOAK) verfügbar. Die Zulassung erfolgte auf Basis der zwei bisher größten Phase III Studien in der Indikation Schlaganfallprophylaxe bei nicht-valvulärem Vorhofflimmern (VHF, ENGAGE-AF) als auch bei der Behandlung von venösen Thromboembolien (VTE, HOKUSAI-VTE). In beiden Zulassungsstudien fand sich bei vergleichbarer Effektivität gegenüber Warfarin eine signifikante Reduktion von Blutungsereignissen gemäß der vordefinierten primären Sicherheitsendpunkte. Zudem ergeben sich aufgrund der einmal täglichen, nahrungsunabhängigen Anwendung, einer eigens untersuchten Strategie zur Dosisreduktion, und dem ansprechenden
\end{abstract}

\footnotetext{
Univ.-Prof. PD Dr. T. W. Weiss, PhD ( $\bowtie) \cdot$ Dr. M. Rohla 3. Medizinische Abteilung, Kardiologie und internistische Intensivmedizin, Wilhelminenspital, Montleartstraße 37, 1160 Wien, Österreich

thomas.weiss@meduniwien.ac.at

Dr. M. Rohla

miklos.rohla@meduniwien.ac.at

Univ.-Prof. PD Dr. T. W. Weiss, PhD

Medizinische Fakultät, Sigmund Freud Universität, Wien, Österreich

PD Dr. B. Dieplinger

Barmherzige Brüder und Barmherzige Schwestern

Zentrallabor, Seilerstätte 4, 4010 Linz, Österreich

benjamin.dieplinger@bs-lab.at

Prof. Dr. H. Domanovits

Universitätsklinik für Notfallmedizin, Allgemeines

Krankenhaus der Stadt Wien, Währinger Gürtel

18-20, 1090 Wien, Österreich

hans.domanovits@meduniwien.ac.at
}

pharmakokinetischen Profil weitere Vorteile in der klinischen Praxis.

Eine interdisziplinäre Expertengruppe nimmt dies zum Anlass, die rezenten Studiendaten und Empfehlungen der nationalen und internationalen Leitlinien in ihrer Kurzform zu skizzieren sowie ein Positionspapier zum Management von Risikopatienten mit nichtvalvulärem VHF sowie der Behandlung von VTE zu formulieren.

Schlüsselwörter Edoxaban · Nicht-VKA Orale Antikoagulanzien · Vorhofflimmern · Venöse Thromboembolien 


\section{Edoxaban for stroke prevention in atrial fibrillation and treatment of venous thromboembolism: an expert position paper}

Summary Edoxaban is the most recent available representative of the Non-Vitamin K antagonist oral anticoagulants (NOAC). The approval was based on the largest phase III trials of NOACs for stroke prevention in patients with non-valvular atrial fibrillation (AF, ENGAGE-AF), and for the treatment of venous thromboembolism (VTE, HOKUSAI-VTE). In both trials, edoxaban was associated with similar efficacy and a significant reduction in bleeding events with respect to the pre-defined primary safety endpoints, as compared to warfarin.

Additionally, the once daily dosing of edoxaban, the clinically investigated strategy for dose-reduction based on clearly defined criteria and the favorable pharmacokinetic profile might further support the clinical applicability of the substance.

In the light of recent data, this expert consensus document aims to summarize the latest clinical trial results while providing a concise overview of current guideline recommendations on the management of patients with non-valvular AF and VTE.

Keywords Edoxaban · Non-Vitamin K antagonist oral anticoagulants · Atrial fibrillation - Venous thromboembolism

\section{Einleitung}

Mit Edoxaban (Lixiana ${ }^{\circledR}$ ) ist nun der neueste Vertreter der Nicht-Vitamin K Antagonisten Oralen Antikoagulanzien (NOAK) zur Schlaganfallprophylaxe bei Patienten mit nicht-valvulärem Vorhofflimmern (VHF) und zur Behandlung und Sekundärprävention venöser Thromboembolien (VTE) in Österreich verfügbar. Eine interdisziplinäre Expertengruppe nimmt dies zum Anlass, die rezenten Studiendaten und Empfehlungen der nationalen und internationalen Leitlinien in ihrer Kurzform zu skizzieren sowie ein Positionspapier zum Management von Risikopatienten mit nichtvalvulärem VHF sowie der Behandlung von VTE zu formulieren. Die für die klinische Anwendung wesentlichen Schlüsselmerkmale von Edoxaban sind in Tab. 1 zusammengefasst.

\section{Epidemiologie}

Nicht-valvuläres VHF ist die häufigste Herzrhythmusstörung und tritt bei 1-2\% der erwachsenen Bevölkerung auf, so dass in Österreich von ca. 120.000 betroffenen Personen auszugehen ist [1].

Die VTE, eine übergeordnete Krankheitsentität, welche die tiefe Venenthrombose (TVT) und Pulmonalembolie (PE) umfasst, ist mit einer Inzidenz von ca. 180/100.000 die dritthäufigste kardiovaskuläre Erkrankung. Dies entspricht ca. 15.500 Fällen in Österreich. Davon entfallen ca. 13.000 auf eine TVT und ca. 2500 auf nicht tödliche, symptomatische PE [2].

\section{Evidenzlage zu den „neuen“ oralen Antikoagulan- zien}

Für alle 4 verfügbaren NOAKs (Apixaban, Dabigatran, Edoxaban und Rivaroxaban), sind qualitativ hochwertige Zulassungsstudien gegenüber einem INR-gesteuerten Vitamin K Antagonisten (VKA) verfügbar [3-11].

Die neuen, im Jahr 2016 veröffentlichten Leitlinien der Europäischen Kardiologischen Gesellschaft (ESC) zum Management von Patienten mit VHF empfehlen Edoxaban als gleichwertige Alternative zu Apixaban, Dabigatran und Rivaroxaban [12].

Obwohl sich die Substanzen in ihrem pharmakologischen Profil unterscheiden und die Zulassungsstudien etwas heterogene Patientenkollektive beleuchtet haben, besteht aufgrund der Größe der Studienkohorten $(n=98,706)$ in der Gesamtheit eine solide, weitgehend homogene Evidenzlage [3-11].

\section{Nicht-valvuläres Vorhofflimmern}

\section{Meta-Analysen der NOAK Studien}

In einer umfassenden Meta-Analyse der Zulassungsstudien aller vier NOAK zeigte sich eine 19-prozentige relative Risikoreduktion von Schlaganfällen oder

\title{
Tab. 1 Schlüsselmerkmale von Edoxaban
}

\author{
In beiden Indikationen - Schlaganfallprophylaxe bei nicht-valvulärem Vorhofflimmern und zur Behandlung von tiefen Venenthrombosen und Lungenembolien \\ sowie Prophylaxe von rezidivierenden tiefen Venenthrombosen und Lungenembolien bei Erwachsenen: \\ Vergleichbare Effektivität gegenüber einem Vitamin K Antagonisten und signifikante Reduktion von Blutungen gemäß der vordefinierten primären Sicherheits- \\ punkte [4, 9] \\ Einmal tägliche Standarddosierung ( $60 \mathrm{mg}$ ) für beide Indikationen (nach mind. 5 tägiger parenteraler Antikoagulation bei venösen Thromboembolien) [4, 9, 31] \\ Für beide Indikationen ist die Dosisreduktion auf $1 \mathrm{x}$ täglich $30 \mathrm{mg}$ klinisch geprüft und bei Vorliegen einer der folgenden Faktoren angezeigt: \\ - errechnete glomeruläre Filtrationsrate von $15-50 \mathrm{ml} / \mathrm{min}$ \\ - Gewicht $\leq 60 \mathrm{~kg}$ \\ - Gemeinsame Anwendung mit Dronedaron, Erythromycin, Ciclosporin oder Ketokonazol. \\ Darunter ist bei erhaltener Effektivität eine tendenziell noch ausgeprägtere Reduktion von Blutungsereignissen gegen über einem Vitamin K Antagonisten zu \\ erwarten $[4,9,31]$ \\ Einnahme unabhängig von den Mahlzeiten [31] \\ Minimales Interaktionspotential aufgrund geringer Metabolisierung über CYP3A4/5 (<10\%). Keine relevanten Interaktionen mit Nahrungsmitteln bekannt [31]
}


Tab. $2 \mathrm{CHA}_{2} \mathrm{DS}_{2}$-VASc-Score zur Risikostratifizierung von Patienten mit nicht-valvulärem Vorhofflimmern

\begin{tabular}{|l|l|l|}
\hline Akronym & Risikofaktor & Punkte \\
\hline C - Congestive Heart Failure & Herzinsuffizienz & 1 \\
\hline H - Hypertension & Arterielle Hypertonie & 1 \\
\hline A - Age $(\geq 75 a)$ & Alter $\geq 75$ Jahre & 2 \\
\hline D - Diabetes & Diabetes mellitus & 1 \\
\hline S-Stroke & Stattgehabter Insult/TIA/Thromboembolie & 2 \\
\hline V-Vascular Disease & Stattgehabter Myokardinfarkt, PAVK, aortaler Plaque & 1 \\
\hline A-Age (65-74a) & Alter 65-74 Jahre & 1 \\
\hline Sc-Sex category & Weibliches Geschlecht & 1 \\
\hline- & Maximalpunkte & 9
\end{tabular}

systemischen Embolien gegenüber VKA (RR 0,81; KI 0,73-0,91), welche hauptsächliche durch eine ausgeprägte Reduktion von hämorrhagischen Schlaganfällen (RR 0,49; $95 \%$ KI 0,38-0,64) bedingt war.

Die Inzidenz gastrointestinaler (GI) Blutungen war unter NOAKs erhöht (RR 1,25; 95 \% KI 1,01-1,55), wobei Apixaban und Dabigatran in der niedrigeren Dosierung $(2 \times 110 \mathrm{mg})$ in diesem Endpunkt eine Ausnahme darstellt $[3-6,13]$.

Auch der indirekte Vergleich schwerer Blutungsereignisse in den einzelnen Studien ergab ein differenziertes Bild: Während für Apixaban, Edoxaban und Dabigatran in der niedrigeren Dosierung $(2 \times 110 \mathrm{mg})$ signifikante Vorteile bei der Vermeidung schwerer Blutungen gegenüber einem VKA gezeigt werden konnten, fand sich kein Vorteil für Dabigatran in der höheren Dosierung $(2 \times 150 \mathrm{mg})$ und für Rivaroxaban [3-6, 13]. In der Meta-Analyse ergab sich daher ein nichtsignifikanter Trend zugunsten von NOAKs gegenüber VKA (RR 0,86; $95 \%$ KI 0,73-1,00, $p=0,06$ ). Das vorteilhafte Sicherheitsprofil der NOAKs lässt sich auch an einer 52-prozentigen Reduktion von intrakraniellen Blutungen (RR 0,48; 95 KI 0,39-0,59) ablesen [13].

\section{Aktuelle Empfehlungen der Leitlinien}

\section{Behandlungsindikation}

In den neuen ESC Leitlinien für das Management von Patienten mit VHF wurde die Indikation zur Antikoagulation anhand des $\mathrm{CHA}_{2} \mathrm{DS}_{2}$-VASc Scores (Tab. 2) neu formuliert, um klar zwischen einer Punktevergabe für das weiblichen Geschlecht und zusätzlichen Risikofaktoren zu unterscheiden [12]. Bei Vorliegen von 2 zusätzlichen Risikofaktoren, d. h. für Frauen mit $\geq 3$ Punkten und Männern mit $\geq 2$ Punkten, ergibt sich eine Klasse IA Empfehlung zur Schlaganfallprophylaxe mit Antikoagulanzien bei nicht-valvulärem VHF. Eine Antikoagulation sollte bei Vorliegen von 2 Punkten (Frauen) oder 1 Punkt (Männer) erwogen werden (Empfehlungsgrad IIaB). Bei Patienten ohne zusätzlichen Risikofaktor (d. h. Männer mit 0 Punkten, Frauen mit 1 Punkt), ist sowohl Antikoagulation als auch Antiplättchentherapie zum Zwecke der Schlaganfallprävention aufgrund des fehlenden klinischen Benefits kontraindiziert (Empfehlungsgrad IIIB) (12).

\section{Wahl der Substanz}

Die österreichische Konsensus-Empfehlung lautet in Übereinstimmung mit den neuen ESC Leitlinien, dass NOAKs aufgrund ihres klinischen Nettonutzens bevorzugt bei Neueinstellungen eingesetzt werden sollen (Empfehlungsgrad IA) [12, 14]. Darüber hinaus ist eine Umstellung immer dann sinnvoll, wenn bei Patienten mit nicht-valvulärem VHF und Indikation zur Antikoagulation aufgrund von schlechter Einstellbarkeit, Nebenwirkungen oder Problemen, die INR-Zielwerte $\mathrm{zu}$ erreichen, VKA nicht angewendet werden können $[14]$.

\section{Acetylsalicylsäure als Alternative zur oralen Antikoagulation?}

Großangelegte retrospektive als auch prospektive Studien konnte zeigen, dass schwere Blutungsereignisse unter Acetylsalicylsäure (ASS) entgegen der subjektiv wahrgenommenen Sicherheit der Substanz vergleichbar häufig aufzutreten scheinen als unter VKA $[15,16]$. In einem indirekten Vergleich einer dänischen Kohortenstudie (118.606 Patienten) wurden tödliche Blutungen und GI-Blutungen numerisch häufiger unter ASS gegenüber VKA beobachtet [16]. Darüber hinaus besteht ein in etwa 50-80\% höheres Risiko für ischämische Ereignisse unter ASS vs. VKA [16, 17].

Die AVERROES Studie randomisierte 5599 Patienten mit nicht-valvulärem VHF und einer Kontraindikation für VKA zu Apixaban vs. ASS. Die Studie wurde aufgrund einer 55-prozentigen Reduktion von Schlaganfällen zugunsten von Apixaban frühzeitig abgebrochen, wobei schwere und intrakranielle Blutungen zwischen den Studienarmen vergleichbar waren [18]. Aus ethischen Überlegungen sind keine derartigen Vergleichsstudien mit den 3 weiteren NOAK verfügbar. Entsprechend ist gemäß den neuen ESC Leitlinien eine Monotherapie mit ASS zum Zwecke der Schlaganfallprävention unabhängig vom Insultrisiko kontraindiziert (Empfehlungsgrad IIIA) (12).

\section{Die richtige Anwendung von Scores zur Einschätzung des Blutungsrisikos}

Die neuen ESC Leitlinien stellen frei welcher Score zur Evaluierung des Blutungsrisiko verwendet werden soll. Diese sollten nicht angewendet werden, um 
über die Indikation oder Beendigung der Antikoagulation zu entscheiden, da Patienten mit erhöhtem Blutungsrisiko ebenfalls ein erhöhtes Schlaganfallrisiko aufweisen und somit überproportional von der Therapie profitieren. Dies konnte in Studien zum „netto klinischen Nutzen“ bestätigt werden [19, 20]. Vielmehr sollten Blutungs-Scores dazu genützt werden, um modifizierbare Risikofaktoren zu erkennen und zu behandeln. In den rezenten Leitlinien sind die Blutdruckeinstellung, die Zeit im therapeutischen Fenster bei VKA, Begleitmedikation wie nicht-steroidale Antirheumatika oder Plättchenaggregationshemmer und starker Alkoholkonsum (>8 alkoholische Getränke pro Woche) als bedeutende modifizierbare Risikofaktoren hervorgehoben [12].

\section{Venöse Thromboembolien}

\section{Meta-Analysen der NOAK Studien}

Bei der Therapie der venösen Thromboembolie (VTE, tiefe Beinvenenthrombose und Pulmonalembolie) wurden für NOAKs unterschiedliche Behandlungsstrategien gewählt und mit der konventionellen Therapie bestehend aus niedermolekularem Heparin (NMH) gefolgt von einem VKA verglichen: Während in den Studien mit Dabigatran und Edoxaban für die Initialbehandlung (ersten Tage) der bisherige Standard mit NMH beibehalten wurde, wurden für Rivaroxaban $(2 \times 15 \mathrm{mg}$ für 21 Tage $)$ und Apixaban $(2 \times 10 \mathrm{mg}$ für 7 Tage) in der akuten Phase höhere Dosierungen eingesetzt. In der sich anschließenden Erhaltungsphase bis 6 Monate werden die gleichen Dosierungen wie bei Patienten mit nicht-valvulärem VHF eingesetzt. Für Apixaban erfolgt - sofern eine Weiterbehandlung über 6 Monate hinaus notwendig ist - eine Dosisreduktion auf $2 \times 2,5 \mathrm{mg}$, während für Edoxaban weiter die Standarddosis von $1 \times 60 \mathrm{mg}$ verwendet werden kann [7-11].

Insgesamt zeigte sich in einer aktuellen Meta-Analyse eine vergleichbare Anzahl an wiederkehrenden VTE oder VTE-assoziierten Todesfällen (RR 0,90, $95 \%$ KI 0,77-1,06) sowie signifikante Vorteile beim Sicherheitsprofil zugunsten der NOAKs, insbesondere bei der Verhinderung von intrakraniellen (RR 0,37, $95 \%$ KI 0,21-0,68) und tödlichen Blutungen (RR 0,36, $95 \%$ KI 0,15-0,84). In Summe traten schwere Blutungskomplikationen unter NOAK vs. VKA um $39 \%$ seltener auf (RR 0,61, $95 \%$ KI 0,45-0,83) [21].

\section{Aktuelle Empfehlungen der Leitlinien}

Wahl des Antikoagulans

In den aktuellen Guidelines des „American College of Chest Physicians“ (ACCP) werden NOAKs zur Behandlung der VTE bei Patienten ohne Tumorerkrankungen als bevorzugte Therapieoption gegenüber VKA empfohlen (Empfehlungsgrad 2B). Bei Patienten mit Tumor-assoziierter Thromboembolie werden nach wie vor $\mathrm{NMH}$ als bevorzugte Therapieoption angesehen (Empfehlungsgrad 2C) [22, 23].

\section{Initiierung der Therapie}

Die US-nationalen ACCP Leitlinien empfehlen für die Standardbehandlung der VTE initial eine parenterale Antikoagulation mit entweder NMH, UFH oder Fondaparinux in jeweils therapeutischer Dosierung [24]. Obwohl eine Zulassung für die komfortablere einmal tägliche Dosierung von NMH $(1,5 \mathrm{mg} / \mathrm{kg}$ Körpergewicht pro Tag) besteht, ist die Datenlage diesbezüglich eingeschränkt, da die Zulassungsstudien für die zweimal tägliche Dosierung ( $1 \mathrm{mg} / \mathrm{kg}$ Körpergewicht alle $12 \mathrm{~h}$ ) durchgeführt worden sind [25]. Im Einklang mit dieser Empfehlung erfolgten die Zulassungsstudien von Dabigatran und Edoxaban unter Beibehaltung der initialen parenteralen Therapie, während Rivaroxaban und Apixaban direkt verabreicht wurden [7-11]. Diese Strategien sind auch in der klinischen Praxis umzusetzen (22).

\section{Dauer der Therapie}

Bei Patienten mit tiefer Beinvenenthrombose oder Pulmonalembolie ist gegenwärtige eine 3-monatige Therapie empfohlen, sofern ein Ereignis vorliegt, das durch einen chirurgischen Eingriff oder einen nichtchirurgischen transienten Risikofaktor ausgelöst wurde (22).

Bei Patienten mit erstmaligem oder wiederholtem Auftreten einer nicht provozierten VTE und niedrigbis moderatem Blutungsrisiko wird eine erweiterte Therapie länger als 3 Monate empfohlen, ohne spezifische Empfehlung hinsichtlich Beendigung der Therapie (d.h. dauerhafte Antikoagulation zur Sekundärprophylaxe aufgrund eines erhöhten Rezidivthromboserisikos) [22].

Diese Empfehlungen werden auch von den positiven Ergebnissen jener NOAK-Studien gestützt, die nach initialer 6-18 monatiger Antikoagulation eine erweiterte Therapie mit NOAK vs. Placebo für weitere 6-12 Monate untersucht haben [22, 26-28]. Im Durchschnitt konnten in den AMPLIFY-EXT (Apixaban), RESONATE (Dabigatran) und EINSTEIN-extension (Rivaroxaban) Studien eine 67-92\% relative Risikoreduktion für wiederkehrende oder tödliche VTE gezeigt werden, wobei schwere Blutungsereignisse nicht häufiger als unter Placebo auftraten [26-28]. Folglich ist aus ethischen Überlegungen eine Placebo-kontrollierte Studie mit Edoxaban nicht verfügbar, jedoch beinhaltete die HOKUSAI-VTE Zulassungsstudie aufgrund ihres Studiendesigns bereits eine Auswertung zur erweiterten Therapie (i. e. Sekundärprophylaxe) gegenüber Warfarin. In dieser post-hoc-Analyse erhielten 7227 Patienten eine verlängerte, 3-12 Monate dauernde Therapie, worunter die Effektivität sowie das Auftreten klinisch relevanter Blutungen mit Edoxaban gegenüber dem VKA vergleichbar waren. Schwere Blutungsereignisse wurden zugunsten von Edoxaban um $55 \%$ reduziert [29]. 
Für Patienten, deren VTE Ereignis in Zusammenhang mit einer Tumorerkrankung steht, wird eine erweiterte Therapie von 3-6 Monaten empfohlen, sofern kein hohes Blutungsrisiko besteht. Danach ist eine individuelle Entscheidung über eine fortführende Sekundärprophylaxe zu treffen [22, 24]. Edoxaban wird gegenwärtig zur Prävention wiederkehrender VTE bei Patienten mit Tumorerkrankung klinisch geprüft (NCT02073682).

Acetylsalicylsäure als Alternative zur oralen Antikoagulation in der Sekundärprophylaxe einer VTE? ASS weist eine weitaus geringere Effektivität zur Vermeidung einer rezidivierenden VTE bei vergleichbarem oder höherem Blutungsrisiko auf. Sofern nach klinischer Abwägung eine erweiterte Therapie indiziert ist, sollten orale Antikoagulanzien bevorzugt eingesetzt werden [22].

\section{Edoxaban, ein neuer Vertreter der NOAKs}

Edoxaban (Lixiana ${ }^{\circledR}$ ) ist der neueste Vertreter der NOAK, dessen Zulassung auf Basis der zwei bisher größten Phase III Studien sowohl in der Indikation Schlaganfallprophylaxe bei nicht-valvulärem VHF (ENGAGE-AF) [4] als auch der Behandlung der VTE (HOKUSAI-VTE) [9] erfolgte. Das pharmakologische Profil von Edoxaban ist insofern ansprechend, als dass die einmal tägliche Dosierung in einer eigens für diesen Zweck durchgeführten Phase II Studie untersucht wurde. Die einmal tägliche Gabe von Edoxaban $60 \mathrm{mg}$ war gegenüber Edoxaban $30 \mathrm{mg} 2 \mathrm{x}$ täglich mit einem tendenziell geringeren Auftreten von schweren oder klinisch relevanten nicht-schweren Blutungen assoziiert [30]. Hervorzuheben ist auch die geringe Metabolisierung über CYP3A4/5 (<10\%) und das demnach minimale Interaktionspotential [31]. Somit ist Edoxaban das einzige NOAK welches die $1 \mathrm{x}$ tägliche Anwendung mit einer nahrungsunabhängigen
Einnahme vereint [31-34]. Lediglich die gemeinsame Anwendung von Edoxaban mit Dronedaron, Erythromycin, Ciclosporin oder Ketokonazol erfordert eine Dosisreduktion auf $30 \mathrm{mg} 1 \mathrm{x}$ täglich. Die Zunahme der Edoxaban Konzentration in Kombination mit Amiodaron, Verapamil oder Chinidin wurde als klinisch nicht signifikant betrachtet [31, 35]. Edoxaban ist in den neuen ESC Leitlinien zum Management von Patienten mit VHF als gleichwertige Alternative zu den bekannten Substanzen Apixaban, Dabigatran und Rivaroxaban empfohlen [12].

Nachfolgend sind die Ergebnisse der Zulassungsstudien von Edoxaban zusammengefasst:

\section{Schlaganfallprophylaxe bei nicht-valvulärem Vor- hofflimmern - Die ENGAGE AF-TIMI 48 Studie}

Die ENGAGE AF-TIMI 48 Studie hat zwischen 2008-2010 insgesamt 21.105 Patienten mit nichtvalvulärem VHF und moderatem bis hohem ischämischen Risiko eingeschlossen. Die Randomisierung erfolgte zu 3 Studienarmen:

- Warfarin (INR 2-3),

- Edoxaban 60/30 mg: 60 mg 1 x tgl., Dosisreduktion auf $30 \mathrm{mg} 1 \times \mathrm{tgl}$,

- Edoxaban 30/15 mg: 30 mg 1 x tgl., Dosisreduktion auf $15 \mathrm{mg} 1 \mathrm{x}$ tgl.

In beiden Edoxaban Studienarmen war die Dosisreduktion von 60 auf $30 \mathrm{mg}$ bzw. von 30 auf $15 \mathrm{mg}$ bei Vorliegen von zumindest einem der folgenden Kriterien vorgesehen:

- eGFR von $30-50 \mathrm{ml} / \mathrm{min}$,

- Körpergewicht $\leq 60$ kg,

- oder Begleittherapie mit Verapamil oder Quinidin.

Die mediane prospektiven Beobachtungszeit betrug 2,8 Jahre (Anmerkung: längste Beobachtungszeit im Vergleich zu den anderen NOAK-Studien).

Das Durchschnittsalter lag bei 72 Jahren, der mittlere $\mathrm{CHADS}_{2}$ Score betrug 2,8 Punkte.
Abb. 1 Vergleichbare Effektivität von Edoxaban gegenüber Warfarin in der ENGAGE-AF Studie

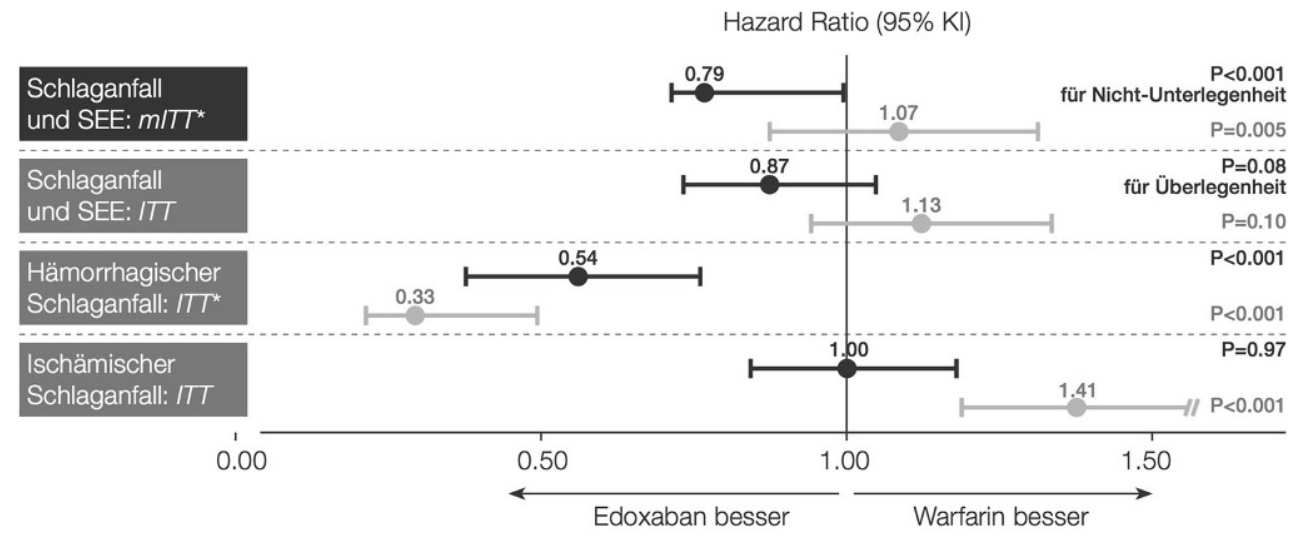

* das verwendete Konfidenzintervall (KI) lag bei $97.5 \%$

** in der modifizierten Intention to Treat Population

† Beinhaltet patientenspezifische Dosisreduktion auf $30 \mathrm{mg} 1 \times$ täglich

‡Beinhaltet patientenspezifische Dosisreduktion auf $15 \mathrm{mg} 1 \times$ täglich

ITT=Intention-to-treat-Analyse; $\mathrm{mIT}=$ =modifizierte Intention-to-treat-Analyse;

$\mathrm{SEE}=$ systemische embolische Ereignisse

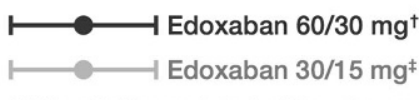

30/15mg Dosierungsstrategie nicht zugelassen 
Abb. 2 Signifikante Reduktion in mehreren Blutungsendpunkten zugunsten von Edoxaban gegenüber Warfarin in der ENGAGE-AF Studie
Abb. 3 Positiver „Netto klinischer Nutzen" zugunsten von Edoxaban gegenüber Warfarin in der ENGAGE-AF Studie

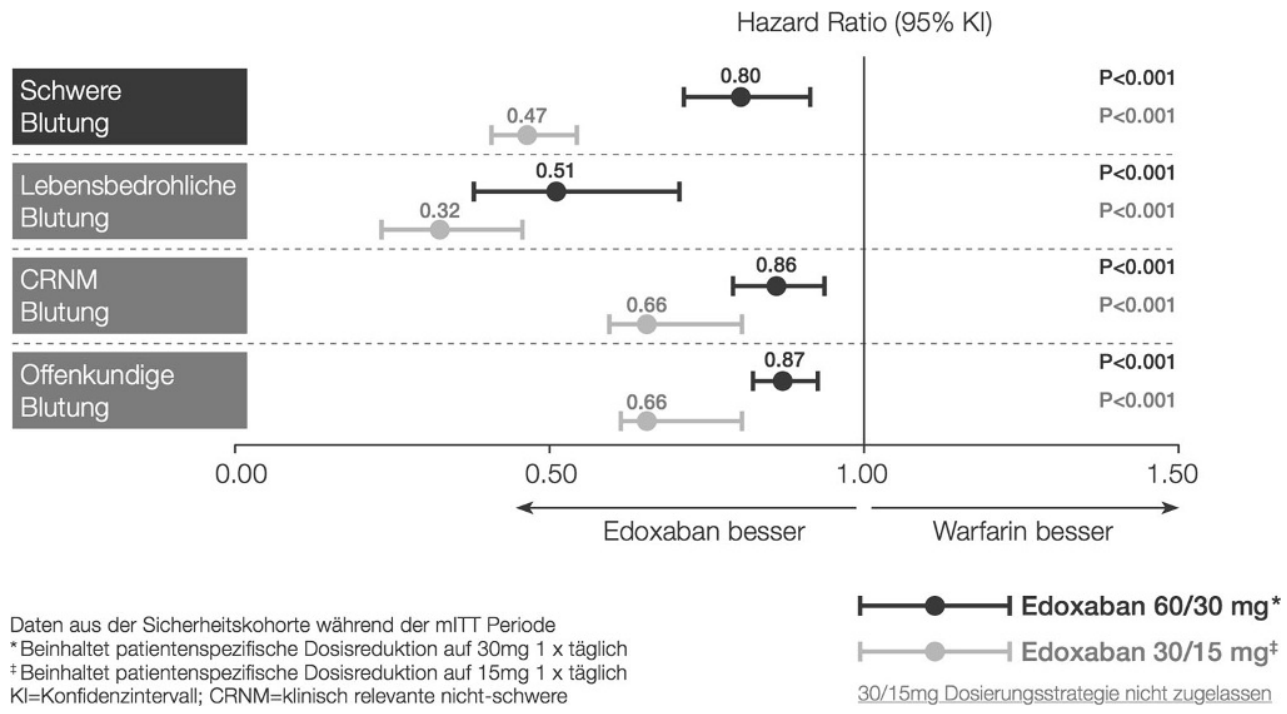

\section{Edoxaban 60/30 mg versus Warfarin}

Der kombinierte Effektivitätsendpunkt, bestehend aus ischämischen oder hämorrhagischen Schlaganfall und systemischer Embolie wurde nach durchschnittlich 2,8 Jahren Follow-Up zugunsten von Edoxaban um $21 \%$ gesenkt (RR 0,79, 97,5\% KI 0,63-0,99, $p<$ 0,001 für Nicht-Unterlegenheit in der modifizierten Intention-to-Treat Population (primärer Endpunkt), RR 0,87, 97,5\% KI 0,73-1,04, $p=0,08$ für Überlegenheit in der Intention-To-Treat Population) (Abb. 1). Hervorzuheben ist die akkurate INR-Einstellung in der Kontrollgruppe, die außerhalb von klinischen Studien selten erreicht wird (median 68,4\% Zeit im therapeutischen Fenster). Der primäre Sicherheitsendpunkt bestehend aus schweren Blutungen wurde zugunsten von Edoxaban um $20 \%$ gesenkt (RR 0,80, $95 \%$ KI 0,71-0,91, $p$ für Überlegenheit $<0,001$ ) (Abb. 2; [4]). Ebenso konnte eine signifikante Reduktion von kardiovaskulären Todesfällen im Edoxaban 60/30 mg
Studienarm verzeichnet werden (RR 0.86, $95 \%$ KI $0,77-0,97, p=0,013$ ), während die Reduktion der Gesamtsterblichkeit statistisch nicht signifikant war (RR 0,92, $95 \%$ KI 0,83-1,01, $p=0,08$ ) [49].

Bei Gegenüberstellung von ischämischen Ereignissen, Blutungsereignissen und der Gesamtsterblichkeit ergab sich für Edoxaban ein positiver „Netto klinischer Nutzen“ (RR 0,89, $95 \%$ KI 0,83-0,96) (Abb. 3; [4]).

\section{Edoxaban 30/15 mg versus Warfarin}

Hinsichtlich des primären Effektivitätsendpunktes war Edoxaban 30/15 mg gegenüber Warfarin nichtunterlegen (RR 1,07, $95 \%$ KI 0,87-1,31, $p=0,005$ ). Schwere Blutungsereignisse wurden in diesem Studienarm noch deutlicher zugunsten von Edoxaban reduziert (RR 0,47, $95 \%$ KI 0,41-0,55, $p<0,001$ ) [4].

Unter diesem niedrigeren Edoxaban Dosierungsschema $(30 / 15 \mathrm{mg})$ traten ischämische Insulte gegenüber Warfarin signifikant häufiger auf (RR 1,41, 95 \% 
Abb. 4 Vergleichbare Effektivität von Edoxaban gegenüber Warfarin in der HOKUSAI-VTE Studie
Abb. 5 Überlegenheit im primären Sicherheitsendpunkt unter Edoxaban gegenüber Warfarin in der HOKUSAI-VTE Studie

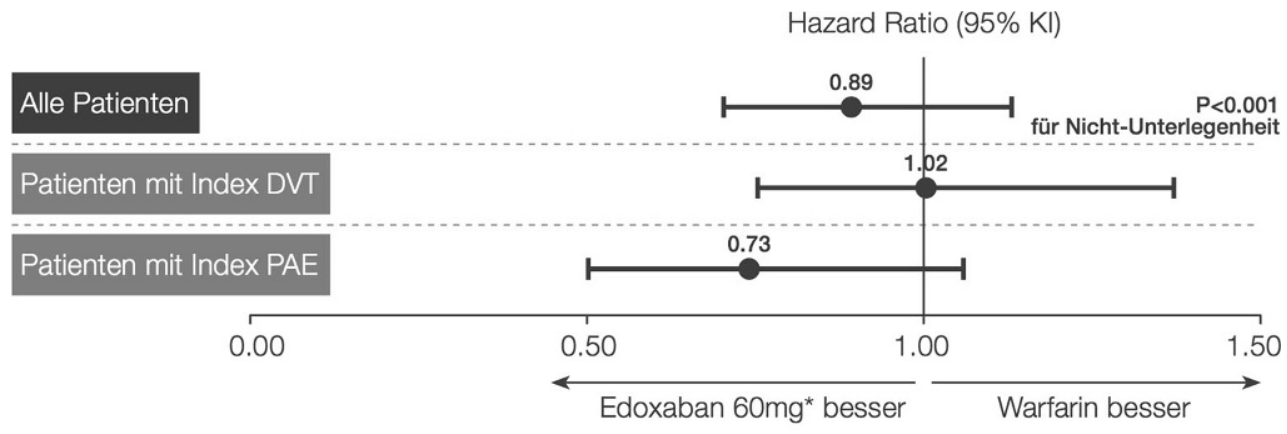

$\mathrm{Kl}=$ Konfidenzintervall; DVT=Tiefe Beinvenenthrombose; PAE=Pulmonalarterienembolie; VTE=venöse Thromboembolie

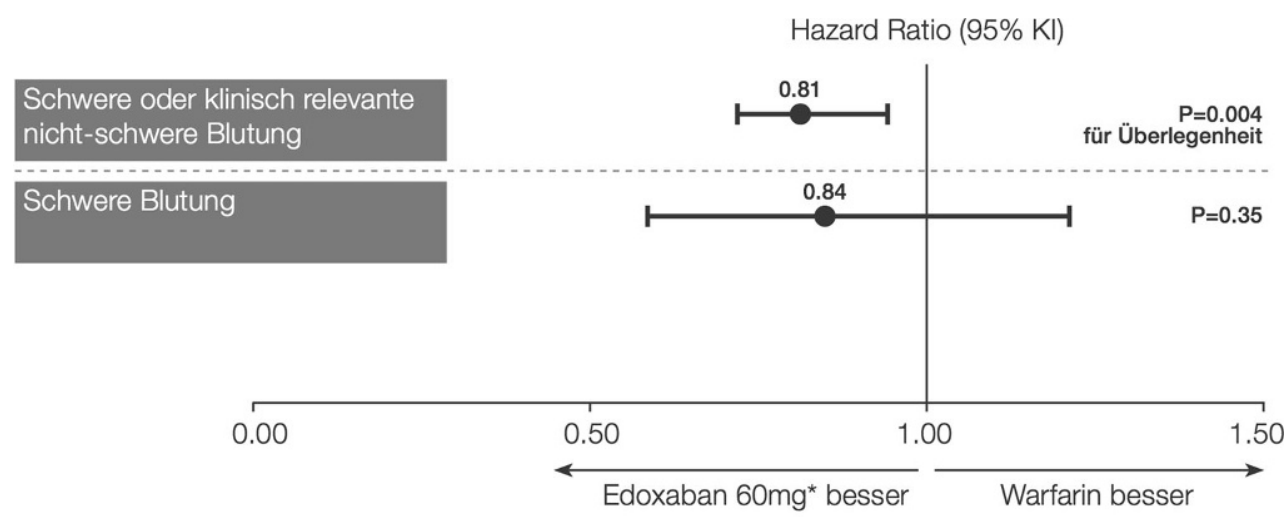

$\mathrm{Kl}=$ Konfidenzinterval

KI 1,19-1,67, $p<0,001)$. Sowohl die Gesamtsterblichkeit (RR 0,87, $95 \%$ KI 0,79-0,96, $p=0,006$ ) als auch kardiovaskuläre Sterblichkeit (RR 0,85, 95 \% KI 0,76-0,96, $p=0,008)$ waren zugunsten von Edoxaban reduziert [4]. Dies ist durch die geringere Inzidenz von schweren Blutungsereignissen zu erklären [49].

Da gegenüber Warfarin eine zumindest vergleichbare Effektivität hinsichtlich der Schlaganfallprophylaxe anzustreben ist, wurde die niedrigere Edoxaban Dosierungsstrategie (30/15 mg) nicht zur Zulassung eingereicht, und wird in der klinischen Praxis nicht verfügbar sein. Ausschließlich zur Transition von Edoxaban $30 \mathrm{mg}$ auf einen VKA ist Edoxaban $15 \mathrm{mg}$ zusammen mit einem VKA anzuwenden. Nachfolgend wird aus diesem Grund ausschließlich auf die Daten des 60/30 mg Studienarmes eingegangen.

\section{Relevante Subgruppen aus der ENGAGE-AF Studie}

\section{Patienten mit Notwendigkeit zur Dosisreduktion}

Die Dosisreduktion von $60 \mathrm{mg}$ auf $30 \mathrm{mg}$ war bei einer eGFR von $30-50 \mathrm{ml} / \mathrm{min}$, Körpergewicht $\leq 60 \mathrm{~kg}$ und Begleittherapie mit Verapamil oder Chinidin vorgesehen, und zwar nicht nur bei Randomisierung sondern auch, wenn einer der Faktoren unter Therapie auftrat. Dies betraf $32,5 \%$ aller Patienten $(25,4 \%$ bei Randomisierung, 7,1\% im Verlauf der Studie).
Bei einer vergleichbaren Rate von Schlaganfällen oder systemischen Embolien (RR 0,81, $95 \%$ KI 0,58-1,13) war die Dosisreduktion mit einer signifikant ausgeprägteren Reduktion von schweren Blutungen unter Edoxaban gegenüber Warfarin assoziiert (RR 0,63, 95\% KI 0,50-0,81, p für Interaktion 0,02 ). Dieser Trend traf auch spezifisch auf Patienten mit einer mäßig reduzierten Nierenfunktion (eGFR 30-50 ml/min) zu (RR 0,75, $95 \%$ KI 0,58-0,98), [4, 31, 36] Patienten mit einer eGFR $<30 \mathrm{ml} / \mathrm{min}$ wurden nicht in die ENGAGE AF-TIMI 48 Studie eingeschlossen. Pharmakokinetische Berechnungen zeigten bei Patienten mit mäßiger (eGFR 30-50 ml/min) und schwerer (eGFR $15-30 \mathrm{ml} / \mathrm{min}$ ) Nierenfunktionseinschränkung eine vergleichbare Zunahme der Edoxaban Plasmakonzentration. Somit besteht eine Zulassung zur Anwendung der reduzierten Dosierung (30 mg 1 x täglich) bis zu einer eGFR von $15 \mathrm{ml} / \mathrm{min}$, jedoch ohne Evidenz aus klinischen Endpunktstudien $[4,31]$.

\section{Sicherheit bei dualer Antiplättchentherapie}

In etwa $30 \%$ der ENGAGE-AF Kohorte erhielt begleitend ASS in prophylaktischer Dosierung (6180 Patienten), was zu einer Verdopplung schwerer Blutungsereignisse in allen Studienarmen geführt hat. Die Reduktion von schweren Blutungsereignissen mit Edoxaban gegenüber Warfarin war unabhängig von 
der begleitenden Antiplättchentherapie (ohne Antiplättchentherapie RR 0,80, $95 \%$ KI 0,68-0,95, mit Antiplättchentherapie RR 0,82, $95 \%$ KI 0,65-1,04, $p$ für Interaktion 0,91). Somit blieb auch der netto klinischen Nutzen von Edoxaban gegenüber Warfarin unabhängig von der begleitenden Antiplättchentherapie erhalten (ohne Antiplättchentherapie RR 0,89, $95 \%$ KI 0,81-0,98, mit Antiplättchentherapie RR 0,82, $95 \%$ KI 0,71-0,95, $p$ für Interaktion 0,35) [37].

\section{Betagte Patienten und Patienten mit Sturzgefahr}

In den prä-spezifizierten Altersgruppen <65, 65-74 und >74 wurde hinsichtlich der primären Effektivitäts- und Sicherheitsendpunkte keine Interaktion beobachtet, somit kann der Vorteil von Edoxaban gegenüber Warfarin in diesen Subgruppen als konsistent betrachtet werden [38]. Aufgrund des naturgemäß höheren Schlaganfall- und Blutungsrisikos bei Patienten über 74 Jahren kam es zu einer größeren absoluten Risikoreduktion von Ereignissen, vor allem von schweren Blutungen, wodurch der „Netto klinische Nutzen“ bei höherem Alter ausgeprägter war. Hinsichtlich des primären netto klinischen Endpunktes bedeutete dies eine 13-prozentige relative Risikoreduktion bei 65-74 Jahren (RR 0,87, 95\% KI 0,76-1,00) und eine 12-prozentige relative Risikoreduktion (RR 0,88, $95 \%$ KI 0,79-0,97) bei >74 Jahren, jeweils zugunsten von Edoxaban [38].

Eine Subgruppe von Patienten mit erhöhten Sturzrisiko, definiert als anamnestizierte Sturzneigung, Schwindel, Schwäche der unteren Extremitäten, kognitives Defizit oder orthostatische Hypotonie wurde ebenfalls beleuchtet. Patienten mit erhöhtem Sturzrisiko hatten unter Edoxaban gegenüber Warfarin eine signifikant größere absolute Risikoreduktion in mehreren Sicherheitsendpunkten, allen voran bei intrakraniellen Blutungen (RR 0,16, $95 \%$ KI 0,04-0,71) und lebensbedrohlichen Blutungen (RR 0,32, $95 \%$ KI 0,10-0,98). Ebenso fand sich eine tendenziell niedrigere Gesamtsterblichkeit zugunsten von Edoxaban [39].

\section{Gastrointestinale Blutungen}

Wie eingangs erwähnt zeigte sich ein konstanter Trend in Richtung erhöhter GI-Blutungen unter NOAKs vs. VKA, mit Ausnahme von Apixaban und Dabigatran in der niedrigeren Dosierung $(2 \times 110 \mathrm{mg})[3-6,13]$. So wurde auch in der ENGAGE AF-TIMI 48 Studie ein 23-prozentiger Anstieg in diesem Endpunkt beobachtet [4]. Lebensbedrohliche oder tödliche GI-Blutungen traten unter Edoxaban jedoch nicht häufiger auf. Insbesondere waren GI-Blutungen unter Edoxaban im Verlauf nicht schwerwiegender als unter Warfarin, wenn man die Notwendigkeit einer chirurgischen Intervention (RR 0,37, $95 \%$ KI 0,16-0,88), einen Hämoglobin-Abfall >5 g/dl (RR 1,01, $95 \%$ KI 0,74-1,38) oder Hospitalisierung (RR 1,14, $95 \%$ KI 0,92-1,40) betrachtet [40].

\section{Therapie von venösen Thromboembolien - Die HOKUSAI-VTE Studie}

Die HOKUSAI-VTE Studie hat zwischen 2010 und 2012 insgesamt 4921 Patienten mit tiefer Venenthrombose (TVT) und 3319 Patienten mit symptomatischer Pulmonalembolie (PE) eingeschlossen. Gemäß USnationaler Leitlinien erhielten in der Akutphase alle Patienten initial für zumindest 5 Tage eine Therapie mit NMH oder UFH. Die Randomisierung erfolgte danach zu Warfarin (INR 2-3) oder Edoxaban $60 \mathrm{mg} 1 \mathrm{x}$ täglich, wobei im Falle einer eingeschränkten eGFR (30-50 ml/min), einem Körpergewicht $\leq 60 \mathrm{~kg}$ oder einer begleitende Therapie mit potenten $P$-Glykoproteininhibitoren (Verapamil, Quinidin) die Dosis auf $30 \mathrm{mg} 1 \mathrm{x}$ täglich reduziert wurde. Es ist an dieser Stelle darauf hinzuweisen, dass damit Edoxaban als einziges NOAK die Möglichkeit einer Dosisreduktion auch in der Behandlung der VTE bietet. Die Dauer der Therapie konnte wie in aktuellen Leitlinien empfohlen nach Abwägung des klinischen Nutzen/Risikoverhältnisses zwischen 3-12 Monaten frei gestaltet werden [22].

Das Durchschnittsalter betrug 56 Jahre, $57 \%$ der Studienteilnehmer waren männlich. Ursächlich für das qualifizierende Ereignis war in $66 \%$ der Fälle eine spontane (nicht-provozierte) VTE, zu $28 \%$ war ein transienter Risikofaktor vorhanden [9]. Die Studie konnte ein breites Patientenkollektiv mit einer unterschiedlich ausgeprägten Thrombuslast bzw. Schweregrad, von einer lokal begrenzten TVT bis zur submassiven PE reichend, einschließen. Edoxaban war im primären Effektivitätsendpunkt, bestehend aus wiederkehrender VTE oder VTE-assoziiertem Tod Warfarin nach 12 Monaten Follow-Up signifikant nicht unterlegen (RR 0,89, $95 \%$ KI 0,70-1,13, $p<0,001$ für Nicht-Unterlegenheit) (Abb. 4). Hinsichtlich des primären Sicherheitsendpunktes (schwere oder klinisch relevante nicht-schwere Blutung) war Edoxaban signifikant überlegen (RR 0,81, 95\% KI 0,71-0,94, $p=$ 0,004 für Überlegenheit) (Abb. 5; [9]).

Patienten, die aufgrund klinischer Parameter eine Dosisreduktion auf $30 \mathrm{mg}$ erhielten, hatten bei erhaltener Effektivität eine signifikante, noch ausgeprägtere Reduktion von klinisch relevanten Blutungsereignissen (RR 0,62, 95 \% KI 0,44-0,86, $p<0,01$ für Überlegenheit) [9, 41]. Eine den Leitlinien entsprechende Therapie über 3 Monate hinaus war ebenso effektiv wie sicher (29).

\section{Relevante Subgruppen aus der HOKUSAI-VTE Studie}

\section{Alter und Körpergewicht}

Jeweils $13 \%$ der in HOKUSAI-VTE eingeschlossenen Patienten waren älter als 75 Jahre oder hatten $\leq 60 \mathrm{~kg}$ Körpergewicht. Die vergleichbare Effektivität von Edoxaban, sowie das überlegene Sicherheitsprofil bei der Reduktion von Blutungsereignissen gegenüber 
Warfarin war unabhängig von Alter und Körpergewicht (9).

\section{Patienten mit Notwendigkeit zur Dosisreduktion}

Die Dosisreduktion von $60 \mathrm{mg}$ auf $30 \mathrm{mg}$ war - analog zur ENGAGE AF-TIMI 48 Studie - bei einer eGFR von $30-50 \mathrm{ml} / \mathrm{min}$, Körpergewicht $\leq 60 \mathrm{~kg}$ und Begleittherapie mit Verapamil oder Chinidin vorgesehen. Dies betraf $18 \%$ der HOKUSAI-VTE Studienpopulation (41).

Im Warfarin Studienarm hatten Patienten die den Dosisreduktionskriterien entsprachen ein um $38 \%$ höheres Risiko einer klinisch relevanten Blutung gegenüber Patienten welche diesen Kriterien nicht entsprachen (RR 1,38, $95 \%$ KI 1,09-1,74).

Im Edoxaban Studienarm konnte durch die Dosisreduktion auf $30 \mathrm{mg}$ gemäß der vordefinierten Kriterien ein Anstieg klinisch relevanter Blutungskomplikationen verhindert werden (RR 0,99, $95 \%$ KI 0,74-1,31). Somit kam es bei Vorliegen von Dosisreduktionskriterien im direkten Vergleich der beiden Substanzen zu einer deutlichen, 38-prozentigen Reduktion relevanter Blutungskomplikationen zugunsten von Edoxaban gegenüber Warfarin (RR 0,62, $95 \%$ KI 0,44-0,86, $p<$ 0,01 für Überlegenheit) [41].

\section{Verlängerte Therapie mit Edoxaban}

Eine Besonderheit der HOKUSAI-VTE stellte die flexible, den Leitlinien und der klinischen Praxis entsprechende Behandlungsdauer von 3-12 Monaten dar, wobei die Beobachtungsdauer unabhängig der Therapiedauer 12 Monate betrug [22, 29]. Dies ist bedeutend da wiederkehrende VTE oftmals kurz nach Absetzen der Therapie auftreten [42]. Bei Patienten die eine Antikoagulation für 3-12 Monate erhielten waren Edoxaban und Warfarin bezüglich des Wiederauftretens von VTE (RR 0,97, 95\% KI 0,7-1,4) und klinisch relevanter Blutungen vergleichbar (RR 0,97, $95 \%$ KI 0,77-1,22). Schwere Blutungen konnten im Zeitraum von 3-12 Monaten unter Edoxaban gegenüber Warfarin signifikant um $55 \%$ reduziert werden (RR 0,45, 95\% 0,22-0,92, $p<0,05$ ). Daher scheint Edoxaban eine attraktive Alternative zu Warfarin in der Sekundärprophylaxe von VTE zu sein [29].

\section{Fragile Patienten}

Die Definition von „fragil“ beinhaltete eine eGFR unter $50 \mathrm{ml} / \mathrm{min}$, Alter über 75 Jahre und Körpergewicht unter $50 \mathrm{~kg}$. Diese Kriterien erfüllten 17 \% des Studienkollektivs. In der Subgruppe von fragilen Patienten fand sich eine signifikante Interaktion bei Betrachtung des primären Effektivitätsendpunktes $(p=0,041)$ zugunsten von Edoxaban, woraus ein tendenziell größerer Nutzen bei fragilen Patienten abgeleitet werden kann. Gleichzeitig wurde das Blutungsrisiko mit Edoxaban vs. Warfarin in einem vergleichbaren Ausmaß reduziert ( $p$ für Interaktion 0,88 ) [43].

\section{Patienten mit Tumorerkrankung}

Aus der HOKUSAI-VTE Studie wurden Patienten mit einer aktiven Krebserkrankung, sofern eine Dauerbehandlung mit NMH indiziert war, ausgeschlossen. Für die Subgruppenanalyse standen 771 Patienten mit anamnestisch bekannter, oder aktiver Tumorerkrankung zur Verfügung. In dieser Subgruppe war die Reduktion von VTE-Rezidiven (RR 0,53, $95 \%$ KI $0,28-1,00)$ und schweren Blutungsereignissen $(0,64$, $95 \%$ KI 0,45-0,92) im Vergleich zur Gesamtkohorte deutlich ausgeprägter [44]. Genauere Daten zu diesem Patientenkollektiv werden gegenwärtig im Rahmen der „HOKUSAI VTE Cancer“ Studie im Vergleich zu einem NMH klinisch geprüft (NCT02073682).

\section{Patienten mit Pulmonalembolie}

In Summe wurden 3319 Patienten mit PE als IndexEreignis eingeschlossen und bei über $90 \%$ dieser Subgruppe wurden NT-pro-BNP Werte bestimmt. Die Ausschüttung von NT-pro-BNP, dem B-Typ natriuretischen Peptid, korreliert mit dem Ausmaß der Rechtsherzbelastung, womit sich dieser Biomarker zur Risikostratifizierung gut eignet [45, 46]. Die Effektivität ( $p$ für Interaktion 0,18 ) und Sicherheit ( $p$ für Interaktion 0,35) von Edoxaban vs. Warfarin war bei Patienten mit und ohne PE konsistent. Bei Patienten, bei denen sich aufgrund stark erhöhter NT-pro-BNP Werte $(\geq 500 \mathrm{pg} / \mathrm{ml})$ eine klinisch bedeutsame Rechtsherzbelastung vermuten lässt, ergab sich mit einer Reduktion von wiederkehrenden VTE ein Vorteil für Edoxaban (RR 0,52, $95 \%$ KI 0,28-0,98) [9, 47].

\section{Zusammenfassung}

Zusammenfassend erfolgte die Zulassung von Edoxaban auf Basis der zwei bisher größten verfügbaren Studien in den jeweiligen Indikationen: In beiden Indikationen - Schlaganfallprophylaxe bei nicht-valvulärem VHF und zur Behandlung von TVT und PE sowie Prophylaxe von rezidivierenden TVT und PE bei Erwachsenen - fand sich bei vergleichbarer Effektivität gegenüber einer gut eingestellten VKA-Therapie eine signifikante Reduktion von Blutungsereignissen gemäß der vordefinierten primären Sicherheitsendpunkte $[4,9$, 20].

Die rezent veröffentlichte ENSURE-AF Studie konnte zeigen, dass Edoxaban bei Patienten mit elektrischer Kardioversion eine sichere sowie effektive Alternative gegenüber einer optimalen Therapie mit NMH und VKA darstellt [48].

In der praktischen Anwendung zeichnet sich die Substanz im Gegensatz zu anderen NOAKs mit einer sowohl einmal täglichen als auch nahrungsunabhängigen fixen Dosierung aus, die bei Vorliegen von klar definierten und in Studien untersuchten klinischen Parametern (eGFR $30-50 \mathrm{ml} / \mathrm{min}$, Körpergewicht $\leq 60 \mathrm{~kg}$, Begleittherapie mit Dronedaron, Erythromycin, Ciclosporin und Ketokonazol) reduziert werden soll [31]. Bei gleichzeitiger Anwendung 
mit Amiodaron, Chinidin oder Verapamil ist keine Dosisreduktion erforderlich [31, 35].

Unter dieser Dosisreduktion von $60 \mathrm{mg}$ auf $30 \mathrm{mg}$ ist kein Anstieg von ischämischen Ereignissen bzw. rezidivierender VTE $\mathrm{zu}$ erwarten, gleichzeitig ist die Reduktion von Blutungsereignissen gegenüber VKA tendenziell ausgeprägter $[4,9,36,41]$. Wie in der ENGAGE AF-TIMI 48 Studie dokumentiert werden konnte, ist eine Unterschreitung dieser Dosierungsstrategie mit einer zusätzlichen Abnahme von schweren, potenziell tödlichen Blutungen vergesellschaftet, jedoch sinkt auch das Potenzial zur Vermeidung von ischämischen Insulten. Die optimale Dosierungsstrategie (Edoxaban 60/30 mg in beiden Indikationen, Schlaganfallprophylaxe bei nicht-valvulärem VHF und Therapie und Prävention von rezidivierender VTE) soll in der klinischen Praxis entsprechend umgesetzt werden $[4,9,31]$.

Zum minimalen Interaktionspotential trägt die geringe und klinisch unbedeutende Metabolisierung über CYP3A4/5 bei [31].

Auf Basis dieses ansprechenden pharmakologischen Profils und der positiven Evidenzlagen wird Edoxaban gegenwärtig bei Patienten mit koronarer Herzerkrankung und Kombination mit einer dualen Antiplättchentherapie (ENTRUST-AF PCI, NCT02866175), und bei Patienten mit Tumor-assoziierter VTE (HOKUSAI-VTE Cancer, NCT02073682) untersucht.

Open access funding provided by Medical University of Vienna.

Interessenkonflikt T.W. Weiss hat Vortrags- und Beratungshonorare von Daiichi Sankyo, Boehringer Ingelheim und Pfizer/BMS erhalten. M. Rohla hat für die Erstellung des Manuskripts ein Honorar von Daiichi Sankyo erhalten. B. Dieplinger hat Vortrags- und Beratungshonorare von Bayer, Boehringer Ingelheim, Bristol-Myers Squibb, Daiichi Sankyo, Pfizer und Roche Diagnostics erhalten. H. Domanovits hat Vortragshonorare von Bayer, Daiichi Sankyo und Boehringer Ingelheim erhalten. D. Fries hat ein Beratungshonorar von Daiichi Sankyo erhalten. M.R. Vosko hat ein Beratungshonorar von Daiichi Sankyo erhalten. T. Gary hat Vortragsund Beratungshonorare von Bayer, Boehringer Ingelheim, Bristol-Myers Squibb, Daiichi Sankyo, Pfizer, Roche Diagnostics, Sanofi Aventis, Medtronic und Aspen Pharma erhalten. C. Ay hat Vortragshonorare von Bayer, Boehringer Ingelheim, Pfizer/BMS und Beratungshonorare von Bayer, Boehringer Ingelheim, Daiichi Sankyo und Pfizer/BMS erhalten.

Open Access Dieser Artikel wird unter der Creative Commons Namensnennung 4.0 International Lizenz (http:// creativecommons.org/licenses/by/4.0/deed.de) veröffentlicht, welche die Nutzung, Vervielfältigung, Bearbeitung, Verbreitung und Wiedergabe in jeglichem Medium und Format erlaubt, sofern Sie den/die ursprünglichen Autor(en) und die Quelle ordnungsgemäß nennen, einen Link zur Creative Commons Lizenz beifügen und angeben, ob Änderungen vorgenommen wurden.

\section{Literatur}

1. Stefansdottir H, Aspelund T, Gudnason V, Arnar DO. Trends in the incidence and prevalence of atrial fibrillation in Iceland and future projections. Europace. 2011;13(8):1110-7.

2. White RH. The epidemiology of venous thromboembolism. Circulation. 2003;107(23 Suppl 1):I4-8.

3. Connolly SJ, Ezekowitz MD, Yusuf S, Eikelboom J, Oldgren J, Parekh A, et al. Dabigatran versus warfarin in patients with atrial fibrillation. NEngl J Med.2009;361(12):1139-51.

4. Giugliano RP, RuffCT, Braunwald E, Murphy SA, Wiviott SD, Halperin JL, et al. Edoxaban versus warfarin in patients with atrial fibrillation. NEngl J Med.2013;369(22):2093-104.

5. Granger CB, Alexander JH, McMurray JJ, Lopes RD, Hylek EM, Hanna M, et al. Apixaban versus warfarin in patients with atrial fibrillation. NEngl J Med.2011;365(11):981-92.

6. Patel MR, Mahaffey KW, Garg J, Pan G, Singer DE, Hacke $\mathrm{W}$, et al. Rivaroxaban versus warfarin in nonvalvular atrial fibrillation. NEngl J Med. 2011;365(10):883-91.

7. Agnelli G, Buller HR, Cohen A, Curto M, Gallus AS, Johnson $\mathrm{M}$, et al. Oral apixaban for the treatment of acute venous thromboembolism. NEngl J Med.2013;369(9):799-808.

8. Bauersachs R, Berkowitz SD, Brenner B, Buller HR, Decousus $\mathrm{H}$, Gallus AS, et al. Oral rivaroxaban for symptomatic venous thromboembolism. N Engl J Med. 2010;363(26):2499-510.

9. Buller HR, Decousus H, Grosso MA, Mercuri M, Middeldorp $\mathrm{S}$, Prins $\mathrm{MH}$, et al. Edoxaban versus warfarin for the treatment of symptomatic venous thromboembolism. N Engl J Med.2013;369(15):1406-15.

10. Buller HR, Prins MH, Lensin AW, Decousus H, Jacobson BF, Minar E, et al. Oral rivaroxaban for the treatment of symptomatic pulmonary embolism. N Engl J Med. 2012;366(14):1287-97.

11. Schulman S, Kearon C, Kakkar AK, Mismetti P, Schellong $\mathrm{S}$, Eriksson $\mathrm{H}$, et al. Dabigatran versus warfarin in the treatment of acute venous thromboembolism. N Engl J Med.2009;361(24):2342-52.

12. Kirchhof P, Benussi S, Kotecha D, Ahlsson A, Atar D, Casadei B, et al. 2016 ESC Guidelines for the management of atrial fibrillation developed in collaboration with EACTS. Eur Heart J. 2016;37(38):2893-962.

13. Ruff CT, Giugliano RP, Braunwald E, Hoffman EB, Deenadayalu N, Ezekowitz MD, et al. Comparison of the efficacy and safety of new oral anticoagulants with warfarin in patients with atrial fibrillation: a meta-analysis of randomised trials. Lancet. 2014;383(9921):955-62.

14. Pabinger I, Lang W, Roithinger FX, Weidinger F, EichingerHasenauer S, Glehr R, et al. Consensus statement: stroke prevention in nonvalvular atrial fibrillation in special consideration of the new direct oral anticoagulants. Wien Klin Wochenschr. 2014;126(23-24):792-808.

15. European Heart Rhythm Association, European Association for Cardio-Thoracic Surgery, Camm AJ, Kirchhof P, Lip GY, Schotten U, et al. Guidelines for the management of atrial fibrillation: the Task Force for the Management of Atrial Fibrillation of the European Society of Cardiology (ESC). Eur HeartJ. 2010;31(19):2369-429.

16. Hansen ML, Sorensen R, Clausen MT, Fog-Petersen ML, Raunso J, Gadsboll N, et al. Risk of bleeding with single, dual, or triple therapy with warfarin, aspirin, and clopidogrel in patients with atrial fibrillation. Arch Intern Med. 2010;170(16):1433-41.

17. Mant J, Hobbs FD, Fletcher K, Roalfe A, Fitzmaurice D, Lip GY, et al. Warfarin versus aspirin for stroke prevention in an elderly community population with atrial fibrillation (the Birmingham Atrial Fibrillation Treatment of the 
Aged Study, BAFTA): a randomised controlled trial. Lancet. 2007;370(9586):493-503.

18. Connolly SJ, Eikelboom J, Joyner C, Diener HC, Hart R, Golitsyn S, et al. Apixaban in patients with atrial fibrillation. NEngl J Med. 2011;364(9):806-17.

19. Banerjee A, Lane DA, Torp-Pedersen C, Lip GY. Net clinical benefit of new oral anticoagulants (dabigatran, rivaroxaban, apixaban) versus no treatment in a „real world" atrial fibrillation population: a modelling analysis based on a nationwide cohort study. Thromb Haemost. 2012;107(3):584-9.

20. Blann AD, Banerjee A, Lane DA, Torp-Pedersen C, Lip GY. Net clinical benefit of edoxaban versus no treatment in a „real world“ atrial fibrillation population: a modelling analysis based on a nationwide cohort study. Int J Cardiol. 2015;201:693-8.

21. van Es N, Coppens M, Schulman S, Middeldorp S, Buller HR. Direct oral anticoagulants compared with vitamin K antagonists for acute venous thromboembolism: evidence from phase 3 trials. Blood. 2014;124(12):1968-75.

22. Kearon C, Akl EA, Ornelas J, Blaivas A, Jimenez D, Bounameaux $\mathrm{H}$, et al. Antithrombotic therapy for VTE disease: CHEST guideline and expert panel report. Chest. 2016;149(2):315-52.

23. Posch F, Konigsbrugge O, Zielinski C, Pabinger I, Ay C. Treatment of venous thromboembolism in patients with cancer: a network meta-analysis comparing efficacy and safety of anticoagulants. Thromb Res. 2015;136(3):582-9.

24. Kearon C, Akl EA, Comerota AJ, Prandoni P, Bounameaux H, Goldhaber SZ, et al. Antithrombotic therapy for VTE disease: antithrombotic therapy and prevention of thrombosis, 9th ed: American College of Chest Physicians evidence-based clinical practice guidelines. Chest. 2012;141(2 Suppl):e419S-94S.

25. Fachinformation Lovenox ${ }^{\circledR} 40 \mathrm{mg}$ Spritzampullen (Stand: Juli2014).

26. Agnelli G, Buller HR, Cohen A, Curto M, Gallus AS, Johnson $\mathrm{M}$, et al. Apixaban for extended treatment of venous thromboembolism. NEngl J Med.2013;368(8):699-708.

27. Romualdi E, Donadini MP, Ageno W. Oral rivaroxaban after symptomatic venous thromboembolism: the continued treatment study (EINSTEIN-extension study). Expert Rev Cardiovasc Ther. 2011;9(7):841-4.

28. Schulman S, Kearon C, Kakkar AK, Schellong S, Eriksson $\mathrm{H}$, Baanstra D, et al. Extended use of dabigatran, warfarin, or placebo in venous thromboembolism. N Engl J Med. 2013;368(8):709-18.

29. Raskob G, Ageno W, Cohen AT, Brekelmans MP, Grosso MA, Segers A, et al. Extended duration of anticoagulation with edoxaban in patients with venous thromboembolism: a post-hoc analysis of the Hokusai-VTE study. Lancet Haematol. 2016;3(5):e228-36.

30. Weitz JI, ConnollySJ, Patel I, Salazar D, RohatagiS, Mendell J, et al. Randomised, parallel-group, multicentre, multinational phase 2 study comparing edoxaban, an oral factorXa inhibitor, with warfarin for stroke prevention in patients with atrial fibrillation. Thromb Haemost. 2010;104(3):633-41.

31. Fachinformation Lixiana ${ }^{\circledR} 60 \mathrm{mg}$ (Stand:August 2016).

32. Fachinformation Pradaxa ${ }^{\circledR} 150 \mathrm{mg}$ Hartkapseln (Stand: Januar 2016).

33. FachinformationXarelto ${ }^{\circledR}$ (Stand: Mai 2016).

34. Fachinformation Eliquis ${ }^{\circledR} 5 \mathrm{mg}$ (Stand:September 2015).

35. Mendell J, Zahir H, Matsushima N, Noveck R, Lee F, Chen $\mathrm{S}$, et al. Drug-drug interaction studies of cardiovascular drugsinvolving P-glycoprotein, an effluxtransporter, on the pharmacokinetics of edoxaban, an oral factor Xa inhibitor. Am JCardiovasc Drugs. 2013;13(5):331-42.

36. RuffCT, Giugliano RP, BraunwaldE, MorrowDA, MurphySA, KuderJF, etal. Association between edoxaban dose, concentration, anti-Factor Xa activity, and outcomes: an analysis of data from the randomised, double-blind ENGAGEAF-TIMI 48 trial. Lancet. 2015;385(9984):2288-95.

37. Xu H, Ruff CT, Giugliano RP, Murphy SA, Nordio F, Patel I, et al. Concomitant use of single antiplatelet therapy with edoxaban or warfarin in patients with atrial fibrillation: analysis from the ENGAGE AF-TIMI48 trial. J Am Heart Assoc. 2016;5(2):e002587.

38. Kato ET, Giugliano RP, Ruff CT, Koretsune Y, Yamashita T, Kiss RG, et al. Efficacy and safety of edoxaban in elderly patients with atrial fibrillation in the ENGAGE AF-TIMI 48 trial.JAm Heart Assoc. 2016;5(5):e003432.

39. Steffel J, Giugliano RP, Braunwald E, Murphy SA, Mercuri M, Choi Y, et al. Edoxaban versus warfarin in atrial fibrillation patients at risk of falling:ENGAGEAF-TIMI 48 analysis. JAm Coll Cardiol.2016;68(11):1169-78.

40. Aisenberg J, Friedman K, Desai J, Weitz JI, Giugliano RP, Ruff CT, et al. Abstract 17392: gastrointestinal bleeding with edoxaban versus warfarin: results from the ENGAGE AFTIMI 48 trial. Circulation. 2015;132:A17392.

41. Verhamme P, Wells PS, Segers A, Ageno W, Brekelmans MP, Cohen AT, et al. Dose reduction of edoxaban preserves efficacy and safety for the treatment of venous thromboembolism. An analysis of the randomised, double-blind HOKUSAIVTE trial. Thromb Haemost. 2016;116(4):747-53.

42. Kearon C, Gent M, Hirsh J, Weitz J, Kovacs MJ, Anderson DR, et al. A comparison of three months of anticoagulation with extendedanticoagulation for afirstepisodeofidiopathicvenous thromboembolism. NEngl J Med. 1999;340(12):901-7.

43. Dobesh PP, Fanikos J. New oral anticoagulants for the treatment of venous thromboembolism: understanding differences and similarities. Drugs. 2014;74(17):2015-32.

44. Raskob GE, van Es N, Segers A, Angchaisuksiri P, Oh D, Boda $Z$, et al. Edoxaban for venous thromboembolism in patients with cancer: results from a non-inferiority subgroup analysis of the Hokusai-VTE randomised, double-blind, doubledummy trial. Lancet Haematol. 2016;3(8):e379-87.

45. Kucher N, Printzen G, Goldhaber SZ. Prognostic role of brain natriuretic peptide in acute pulmonary embolism. Circulation. 2003;107(20):2545-7.

46. Sanchez O, Trinquart L, Colombet I, Durieux P, Huisman MV, Chatellier G, et al. Prognostic value of right ventricular dysfunction in patients with haemodynamically stable pulmonary embolism: a systematic review. Eur Heart J. 2008;29(12):1569-77.

47. Brekelmans MP, Ageno W, Beenen LF, Brenner B, Buller $\mathrm{HR}$, Chen CZ, et al. Recurrent venous thromboembolism in patients with pulmonary embolism and right ventricular dysfunction: a post-hoc analysis of the Hokusai-VTE study. LancetHaematol.2016;3(9):e437-45.

48. Goette A, Merino JL, Ezekowitz MD, Zamoryakhin D, Melino M, Jin J, et al. Edoxaban versus enoxaparin-warfarin in patients undergoing cardioversion of atrial fibrillation (ENSURE-AF): a randomised, open-label, phase $3 \mathrm{~b}$ trial. Lancet. 2016;388(10055):1995-2003. doi:10.1016/s01406736(16)31474-x.

49. Giugliano RP, Ruff CT, Wiviott SD, Nordio F, Murphy SA, Kappelhof JAN, et al. Mortality in patients with atrial fibrillation randomized to edoxaban or warfarin: insights from the ENGAGE AF-TIMI 48 Trial. Am J Med. 2016;129(8):850857.e2 